\title{
Clinically Important Misclassification of Aortic Valve Stenosis Severity Using Non- Invasive Techniques: Simultaneous Echocardiography and Cardiac Catheterization During Transcatheter Aortic Valve Implantation in Awake Patients
}

\author{
Pranav Loyalka, MD,${ }^{1}$ Faisal H. Cheema, MD,${ }^{1,2,3}$ Pratap Singh, MBBS,${ }^{4}$ Charles C. Miller III, PhD, ${ }^{5}$ \\ Roy Sheinbaum, MD ${ }^{6}$ Anthony L. Estrera, MD, ${ }^{5}$ Hazim J. Safi, MD, Keshava Rajagopal, MD, PhD ${ }^{1,3}$ \\ ${ }^{1}$ Houston Heart, HCA Houston Healthcare, Houston, TX, USA; ${ }^{2}$ HCA Research Institute, Nashville, TN, USA; ${ }^{3}$ University of \\ Houston College of Medicine, Houston, TX, USA; ${ }^{4}$ Division of Cardiology, Department of Internal Medicine; ${ }^{5}$ Department of \\ Cardiovascular and Thoracic Surgery; ${ }^{6}$ Department of Anesthesiology; University of Texas-Houston McGovern Medical School, \\ Houston, TX, USA
}

\section{ABSTRACT}

Background: Suitability for transcatheter aortic valve (AV) implantation (TAVI) is determined by using transthoracic echocardiography (TTE), although left-sided cardiac catheterization (LCC) provides directly measured pressure data. TAVI in awake patients permits simultaneous comparison of TTE and LCC under physiologically relevant left ventricular loading conditions. We hypothesized that clinically important discrepancies between TTE and LCC would be identified.

Methods and results: TAVI was performed in 108 awake patients undergoing intra-procedural TTE and LCC between January 1, 2016 and December 31, 2016, based upon preprocedure TTE data. Intra-procedural assessments simultaneously were performed before and after prosthesis implantation. Based upon mean trans-AV systolic ejection pressure gradient (MSEPG), AS was graded as: mild ( $<20 \mathrm{~mm} \mathrm{Hg}$; grade 1), moderate (20 - <40 mm Hg; grade 2), or severe ( $\geq 40$ $\mathrm{mm} \mathrm{Hg}$; grade 3). In 79 of the $108(73.1 \%)$ patients, intraprocedural TTE and LCC assessments were concordant. In 2 of the $108(1.9 \%)$ patients, TTE overestimated AS severity by $\geq 1$ grade. In 27 of the 108 (25.0\%) patients, TTE underestimated AS severity by $\geq 1$ grade. In total, AS severity reclassification occurred in 29 (26.9\%) patients. Overall, TTE underestimated MSEPG by $8.9 \pm 1.2 \mathrm{~mm} \mathrm{Hg}$ (TTE MSEPG versus LCC MSEPG; $P<.001)$.

Conclusion: Current TTE criteria appear to frequently and importantly underestimate AS severity. Because decisionmaking regarding TAVI often exclusively is based upon TTE data, these findings suggest either a continued role for LCC in the diagnostic assessment of AS in patients who do not meet standard TTE criteria or lowering TTE cutoffs for TAVI.

Received Fune 11, 2020; accepted August 5, 2020.

Correspondence: Keshava Rajagopal, $M D, P b D$, Department of Clinical Sciences, University of Houston College of Medicine, and Houston Heart, HCA Houston Healthcare; 1200 Binz Street, Suite 900, Houston, TX, USA; 713-522-0220 (e-mail:krajago2@central.uh.edu)
Highlights:

- TTE is the benchmark standard methodology for AS assessment prior to TAVI

- Simultaneous TTE and catheterization assessments of AS were compared prior to TAVI

- TTE frequently and importantly underestimated AS severity relative to catheterization

- In patients suspicious for severe AS, if TTE data identifies less than severe AS, pre-TAVI catheterization may be valuable

\section{INTRODUCTION}

Aortic valve (AV) stenosis (AS) is the most or second most common form of valvular heart disease [Andell 2017]. Classic studies have demonstrated the aggressive and malignant natural history of symptomatic and severe AS [Braunwald 2018]. As a mechanical disease in which left ventricular (LV) outflow during systolic ejection is impeded, mechanical strategies are the cornerstone of therapy for hemodynamically important severe AS. Historically, open cardiac surgical aortic valve replacement (AVR) has been the standard treatment of severe AS, with a wide range of artificial mechanical and biological (stented xeno-prostheses, stentless xeno- and allo-prostheses, and autografts) prosthetic options [Reineke 2016]. Over the past two decades, however, transcatheter aortic valve implantation (TAVI), also termed transcatheter aortic valve replacement (TAVR), has emerged as an alternative mechanical therapy for AS. In high and intermediate surgical risk patients, TAVI provides effective treatment of AS, with superior periprocedural mortality and morbidity compared with AVR [Leon 2010; Smith 2011; Popma 2014; Adams 2014].

Regardless of whether AVR or TAVI is chosen as the treatment strategy for AS, accurate assessment of the physiological severity of AS is vitally important. Underestimating AS severity will result in under-treatment of patients; overestimating AS severity, in contrast, will result in over-treatment of patients. Assessment of the pressure gradients between the LV and proximal aorta during LV systolic ejection - either 


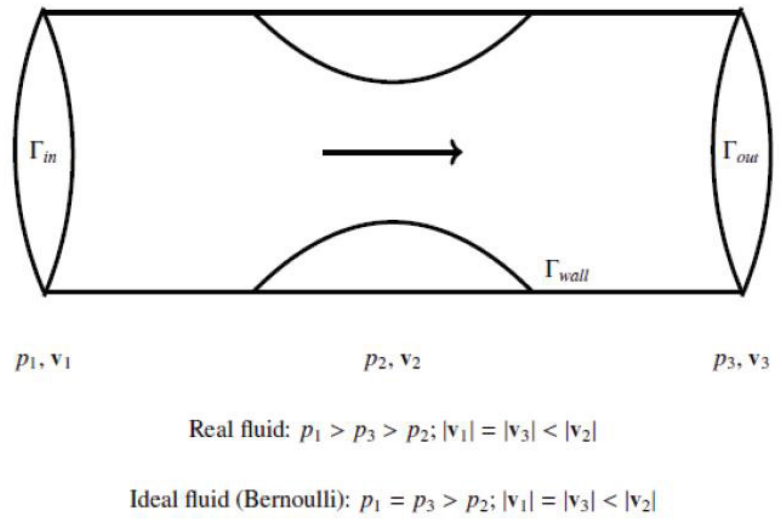

Forced Application of Ideal fluid to Real fluid: $p_{1}>p_{3}=p_{2} ;\left|\mathbf{v}_{1}\right|=\left|\mathbf{v}_{3}\right|<\left|\mathbf{v}_{2}\right|$

Figure 1. Depiction of fluid mechanics of AS, demonstrating assumptions and conceptual flaws in conventional Bernoulli equation-based non-invasive calculation of transvalvular pressure gradients. Modified from Svihlova et al, terms are pressure ( $p)$, velocity $(v)$, and cross-sectional area $(\Gamma)$ [Svihlova 2017]. Zones 1, 2, and 3, refer to the inlet, stenosis, and outlet, with the arrow indicating the direction of flow. Catheterization-measured findings are what are observed under the first heading of "real fluid." The inlet pressure $\mathrm{p} 1$ is $>$ the outlet pressure $p 3$, with the difference across the stenosis $(p 1-p 3)$ being what is clinically termed the "pressure gradient." The intra-stenosis pressure p2 is the lowest value, and typically is some amount lower than p3. Energy is dissipated and expressed in the $(p 1-p 3)$ pressure gradient. The second, "ideal fluid," constitutes the circumstance under which the Bernoulli equation holds. The inlet pressure $\mathrm{p} 1$ is the highest value, and inlet velocity $\mathrm{v} 1$ is the lowest value. However, if the cross-sectional area of the inlet and outlet are identical without alternate flow paths (single conduit), then the inlet and outlet pressures are the same ( $\mathrm{p} 1$ $=p 3)$, and the inlet and outlet velocities are the same $(v 1=v 3)$. No energy dissipation occurs. Within the stenosis, velocity increases and pressure decreases; if the Bernoulli equation holds, then the extent of velocity increase and pressure decrease occur in order to satisfy $p$ $+(\rho \vee 2 / 2)+\rho g h=$ constant, where $\rho=$ fluid density and $h=$ height relative to baseline (this is assumed to be constant for the purposes of this study). The first term is the pressure component of potential energy, the second term is kinetic energy, and the third term is gravitational potential energy. Echocardiography calculations fall under the third heading of "forced application of ideal fluid to real fluid." The Bernoulli equation is used, but does not actually hold true across the entire length of the conduit even in the forced application. Here, p1 still is the highest value, with $p 2=p 3$, but both lower than $p 1$. Because the Bernoulli equation holds in Zones 1 and 2, the degree to which $v$ increases from $v 1$ to $v 2$ can be used to calculate the degree to which $p$ decreases from $p 1$ to $p 2$. However, the entirety of the pressure decrease within the stenosis is held constant into Zone 3. Consequently, the trans-stenosis pressure difference $(p 1-p 3)$ happens to be equal to $(p 1-p 2)$, but $v 3$ is still lower than $v 2$, and equal to $v 1$. There is no formal rational basis for this, however. Specific mention is made of three additional points. First, conservation of mass (the "continuity equation") holds under all circumstances. This is why $v 1$ and $v 3$ must be equal under both ideal and real fluids circumstances, if the cross-sectional areas at the inlet and outlet are identical, with no alternate flow paths (single conduit). Second, "pressure recovery," namely $\mathrm{p} 3$ being some amount higher than $\mathrm{p} 2$, commonly occurs under real circumstances, but is assumed not to occur in non-invasive assessments. In non-invasive assessment via Bernoulli equation-based methods, p2, the pressure within the stenosis, is conveniently assumed to be equal to $\mathrm{p} 3$, the pressure downstream of the stenosis. In contrast, pressure recovery is partial under real circumstances, and total under ideal circumstances. Third, it may seem that non-invasive approaches thus ought to systematically overestimate trans-stenosis pressure drop, rather than mis-estimate via underestimation. However, systemic underestimation would mean that the Bernoulli equationcalculated value of $\mathrm{p} 2$ (which equals $\mathrm{p} 3$ in the calculated model) is consistently lower than the measured value of $\mathrm{p} 3$, even if one were to fix identical values for $\mathrm{p} 1$ between calculated and measured circumstances. There is no rational basis for this. In non-invasive assessment, within the stenosis, the amount by which kinetic energy increases necessarily must be balanced by the amount that potential energy (pressure) decreases, because energy is conserved in this segment since the Bernoulli equation - which expresses conservation of energy - is assumed to be true. All of the energy losses for the real fluid "forced" to operate under ideal circumstances within Zones 1 and 2 are assumed to occur in Zone 3, such that all of the fluid energy lost across the stenosis can be expressed in kinetic energy losses (velocity decrease in the poststenotic segment). In contrast, for a real fluid, with measurements undertaken via catheterization, the decrease in $\mathrm{p} 2$ conceptually is not constrained by the increase in $\mathrm{v} 2$, because total energy is not conserved, i.e., energy dissipation may occur such that the real $p 2$, from which pressure increases to the real $\mathrm{p} 3$ to an extent determined by what $\mathrm{v} 3$ must be as dictated by conservation of mass, is much less than the idealized Bernoulli equation-based p2 (which equals the idealized p3).

by measurement or calculation [Svihlova 2017], or calculation of "valve resistance" (impedance, in the context of unsteady flow) [Ford 1990], are the best-known methods to determine the physiological severity of AS. In contrast, AV area (AVA) determination relates to the anatomic severity of AS.

Transthoracic echocardiography (TTE) and left-sided cardiac catheterization (LCC) are the most common approaches to assess AS prior to TAVI. Current criteria for TAVI are specifically TTE-based (and are physiologic as opposed to anatomic, with the exception of AVA), as outlined in the 2017 American College of Cardiology Expert Consensus Decision Pathway [Otto 2017]. However, LCC measures pressure, whereas all non-invasive assessments calculate pressure. Moreover, the theoretical bases for such calculations used to determine physiological stenosis severity have limitations (Figure 1). Most importantly, LCC-measured and TTE-calculated trans-AV systolic ejection pressure gradients long have been known to have substantial discrepancies. Oh et al [Oh 1988], in an early TTE study from the Mayo Clinic, found that TTE had only a $40-50 \%$ sensitivity in the identification of severe AS, with underestimation of trans-AV systolic ejection pressure gradients. Conversely, with respect to anatomic severity of AS, TTE can measure or calculate AVA, whereas LCC only calculates AVA. Recently, Gertz et al demonstrated poor correlation between TTE- and LCCdetermined AVA area [Gertz 2012]. Consequently, exclusive reliance upon TTE to determine appropriateness for TAVI, particularly with respect to physiologic parameters that are calculated rather than measured, may result in clinically important inaccuracies.

Awake TAVI [Lauck 2014] provides physiologically more relevant and uniform hemodynamic and LV loading conditions under which AS severity can be assessed simultaneously 
using LCC and TTE, in contrast to general anesthesia. The empirical validity of standard TTE approaches to calculating trans-AV pressure gradients thus can be directly compared with LCC. We examined an institutional series of awake transfemoral TAVI, in order to directly compare LCC and conventional TTE assessments and identify any discrepancies with regard to AS severity.

\section{METHODS}

Overview and patients: A series of 108 consecutive patients undergoing awake transfemoral TAVI and simultaneous intra-procedural LCC and TTE performed by a single interventional cardiologist (PL) at Memorial Hermann-Texas Medical Center between January 1, 2016 and December 31, 2016, were analyzed. All patients underwent pre-TAVI TTE. Intra-procedural TTE was interpreted in a blinded fashion. Severe AS was determined based upon a peak trans-AV systolic ejection velocity $\geq 4.0 \mathrm{~m} / \mathrm{s}$ [corresponding to a peak instantaneous trans-AV systolic pressure gradient (PISEPG) $\geq 64 \mathrm{~mm} \mathrm{Hg}]$ and/or a calculated mean trans-AV systolic ejection pressure gradient (MSEPG) of $\geq 40 \mathrm{~mm} \mathrm{Hg}$ (calculation described below). Of the patient group, 106 had severe AS based upon these criteria. Two patients had predominant AV regurgitation (AR) as an off-label indication for TAVI, one of whom did not have AS; both were included. This cohort of patients corresponds to the initial experience of our group with awake TAVI.

TAVI procedure, TTE and LCC: Monitored anesthesia care (MAC) was used for all procedures. Intravenous anesthetic agents, comprised of either propofol or dexmedetomidine (the latter only used in patients with preserved LV systolic function), along with small amounts of fentanyl as needed, were used in concert with local anesthetic agents ( $2 \%$ lidocaine administered subcutaneously) at vascular access sites. All procedures in this series were transfemoral TAVI using the Edwards Sapien TAVI system (Edwards Lifesciences, Irvine, CA).

Bilateral common femoral arterial and venous access was obtained, typically percutaneously; when deemed clinically warranted, open femoral arterial access was obtained. The more anatomically suitable femoral artery was used for largebore sheath placement, through which retrograde trans-AV aortic root-to-LV access was obtained. Simultaneous LV and ascending thoracic aortic pressures were measured using the newly placed $\mathrm{LV}$ catheter and a secondary aortic catheter ( $\mathrm{LV}$ catheter $6 \mathrm{Fr}=2.00 \mathrm{~mm}$, aortic catheter $5 \mathrm{Fr}=1.67 \mathrm{~mm}$ diameter) as functions of time. MSEPG and peak-to-peak systolic ejection pressure gradients (PPSEPG) were determined, but PISEPG was not recorded. MSEPG was computed via catheterization laboratory software using the definition of the mean values of functions in calculus, integrating through the systolic ejection time, from $A V$ opening to closure: $\left[\int\left[\mathrm{P}_{L V}(t)\right.\right.$ $\left.\left.-\mathrm{P}_{\text {aorta }}(\mathrm{t})\right] \mathrm{dt}\right] / \mathrm{t}_{\text {systolic ejection }}$. TTE was performed simultaneously. Long- and short-axis views of the AV were obtained, and velocities in the $\mathrm{LV}$ outflow tract and aortic valve orifice/aortic root were measured as functions of time. Trans-AV
PGs were calculated from these velocity data using the modified Bernoulli equation $\left[\Delta \mathrm{P}_{\mathrm{IV}-\mathrm{arra}}=4 \mathrm{v}_{\mathrm{AV}}{ }^{2}\right.$; the PISEPG was determined using the peak systolic ejection velocity, whereas the MSEPG was determined using the mean systolic ejection velocity, in turn determined by numerically calculating the velocity-time integral (VTI) during systolic ejection, and dividing the VTI by the systolic ejection time]. Thereafter, the $L V$ catheter was withdrawn over a guidewire, and the valve prosthesis mounted onto the delivery system was advanced retrograde through the systemic arterial circulation, until the valve prosthesis was in an intra-annular position. Rapid ventricular pacing was initiated, and the valve was deployed. Thereafter, the delivery system was withdrawn antegrade, and a pressure-transducing catheter was advanced over the guidewire until it resided within the $\mathrm{LV}$ cavity. Repeat $\mathrm{AV}$ assessments by LCC and TTE were performed as described. Based upon MSEPG, AS was graded as: mild ( $<20 \mathrm{~mm} \mathrm{Hg}$; grade 1), moderate (20 - <40 $\mathrm{mm} \mathrm{Hg}$; grade 2$)$, or severe ( $>40$ $\mathrm{mm} \mathrm{Hg}$; grade 3).

Data analysis: Comparisons were made between simultaneously obtained intra-procedural TTE and LCC data, with only intra-patient comparisons being performed. Consequently, paired Student's t-tests were used to compare LCC and TTE data. The Receiver Operator Characteristic (ROC) curves were used to analyze the diagnostic accuracy of TTE vs. LCC derived AV assessments intra-procedurally. The area under curve (AUC) ranges from 0 to 1. TTE that correctly classifies all AV assessments based on the gold-standard LCC will have an AUC of 1.0, and a TTE with an AUC of 0.5 or less will have no discriminatory value. Data were analyzed using SPSS Version 25 (IBM, Armonk, NY).

\section{RESULTS}

Patient characteristics and peri-procedural TAVI outcomes: As stated in Methods, 108 patients underwent awake transfemoral TAVI with simultaneous LCC and TTE assessments performed pre- and post-AV prosthesis implantation. Native valve TAVI accounted for 107 cases, while valve-in-valve TAVI accounted for one case. Hospital mortality was $0.9 \%$ (one out of 108). Median length of stay was two days. Complications assessed included: need for emergent open cardiac surgery, shock requiring institution of mechanical circulatory support (typically peripheral veno-arterial extracorporeal membrane oxygenation), major bleeding ( $>4$ units of exogenous blood products), lower extremity ischemic complications, and pacemaker implantation. These data are displayed in Table 1.

Intra-procedural LCC and TTE assessments of AS severity: We assessed the correlation between pre-implantation LCC and TTE assessments of AS. As stated in Methods, LCC determined MSEPG and PPSEPG, but not PISEPG. In contrast, TTE calculated both PISEPG and MSEPG. Consequently, LCC-assessed MSEPG and PPSEPG were compared only with TTE-assessed MSEPG. As shown in Figure 2 and Table 2(A), TTE MSEPG was substantially and significantly lower than LCC MSEPG, as well as LCC PPSEPG. The mean difference between TTE and LCC MSEPG was $-8.9 \pm 1.2$ 


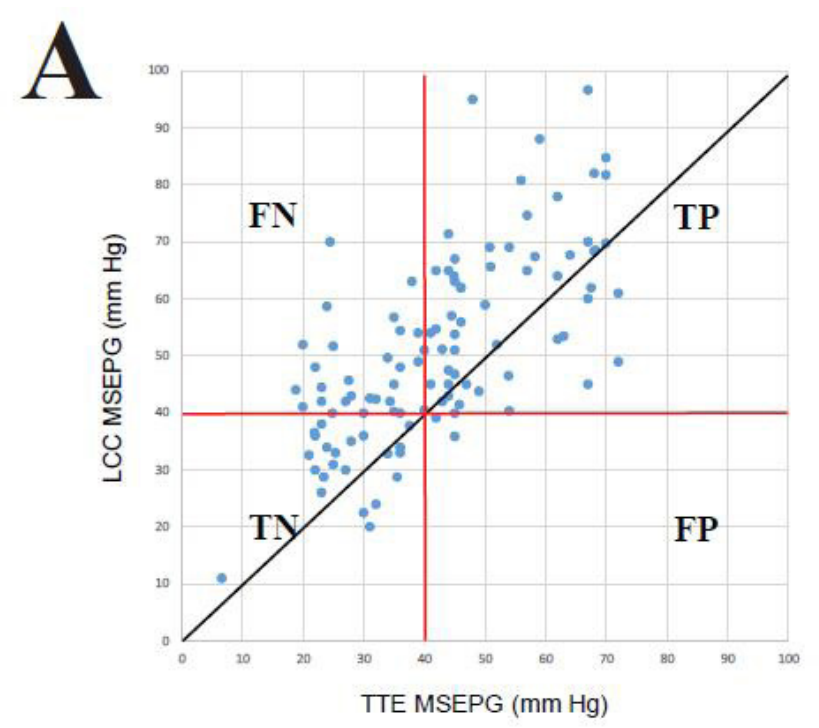

B

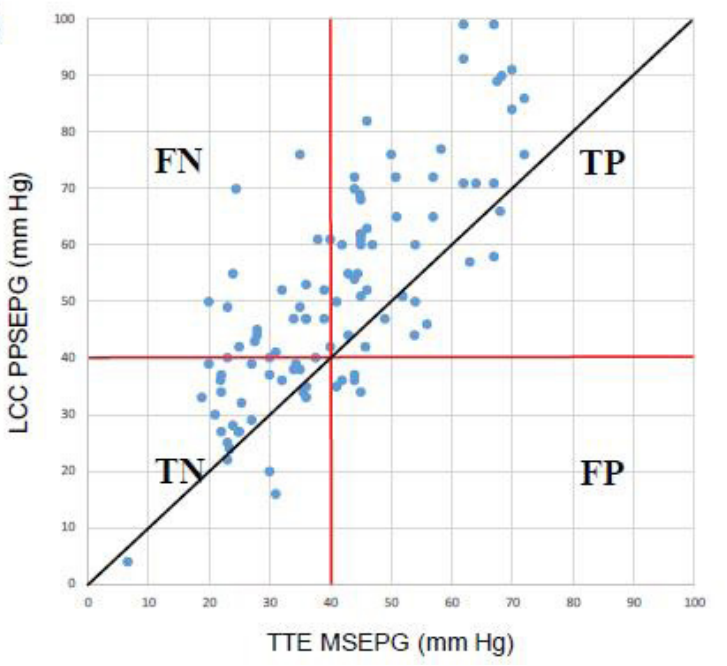

Figure 2. Graphs of LCC-derived versus TTE-derived SEPG, with TTE assessment resulting in underestimation relative to LCC. A) LCC MSEPG ( $y$-axis) versus TTE MSEPG ( $x$-axis). B) LCC PPSEPG ( $y$-axis) versus TTE MSEPG ( $x$-axis). The line $y=x$ denotes ideal correlation between LCC and TTE; values above the line denote underestimation by TTE, while values below the lines denote overestimation by TTE. The red lines indicate $40 \mathrm{~mm} \mathrm{Hg} \mathrm{PGs,} \mathrm{the} \mathrm{cutoff} \mathrm{value} \mathrm{for} \mathrm{severe} \mathrm{stenosis.} \mathrm{True}$ and false positive and negative data are relative to this value.

(SEM) $\mathrm{mm} \mathrm{Hg}(P<.001)$, while the mean difference between TTE MSEPG and LCC PPSEPG was $-13.3 \pm 1.8($ SEM) $\mathrm{mm}$ $\mathrm{Hg}(P<.001)$ (Figure 2 and Table 2$)$.

Although TTE calculations underestimated LCC measurements, since all patients were thought to have severe AS based upon pre-TAVI TTE, the clinical consequences of this underestimation were not clear. We assessed whether the intra-procedural TTE estimates would result in different classifications of AS severity in comparison to LCC. As shown in Figure 2 and Table 2(B), TTE underestimation of AS severity resulted in a substantial degree of severity misclassification.
Table 1. Demographic and clinical outcomes data for patients undergoing awake transfemoral TAVI with simultaneous LCC and TTE assessment of AS. Periprocedural mortality is defined as index hospitalization mortality. Need for emergent cardiac operation is defined as an operation on the heart and/ or great vessels, excluding percutaneous procedures such as pericardiocentesis. Mechanical circulatory/pulmonary support includes de novo (intra- or post-procedural) intra-aortic balloon pump placement, percutaneous/endovascular or surgical ventricular assist device placement, and percutaneous/ endovascular or surgical cannulation for extracorporeal membrane oxygenation. Major bleeding is defined as transfusion of $\geq 4$ units of exogenous blood products.

\begin{tabular}{lc}
\hline Details & $\mathrm{N}(\%)$ \\
\hline Total number of patients: 108 & \\
Native valve TAVI & $107(99.1 \%)$ \\
Valve-in-valve TAVI & $1(0.9 \%)$ \\
Peri-procedural mortality & $1(0.9 \%)$ \\
Median length of stay \pm SD (days) & $2 \pm 3.5$ \\
Need for emergent cardiac surgery & $0(0 \%)$ \\
Peri-procedural mechanical circulatory support & $1(0.9 \%)$ \\
Major bleeding & $2(1.9 \%)$ \\
Lower extremity ischemia & $4(3.7 \%)$ \\
Surgical femoral arterial repair & $11(10.2 \%)$ \\
Pacemaker implantation & $8(7.4 \%)$
\end{tabular}

Of 108 patients, based upon MSEPG, LCC identified severe AS in 84 patients, moderate AS in 23 patients, and mild AS in one patient. TTE identified severe AS in 59 patients, moderate AS in 47 patients, and mild AS in two patients. By examining intra-patient comparisons between LCC and TTE, AS severity was found to be concordant in 79 of the $108(79 / 108$ $=73.1 \%)$ patients. Of the 29 cases $(29 / 108=26.9 \%)$ of discordant data, TTE overestimated AS severity by $\geq 1$ grade in two patients $(2 / 108=1.9 \%)$. In contrast, TTE underestimated AS severity by $\geq 1$ grade in 27 patients $(27 / 108=25.0 \%)$.

Similar findings were observed for TTE MSEPG in comparison to the LCC PPSEPG (Figure 2 and Table 2(B). Of 108 patients, based upon PPSEPG (using MSEPG criteria), LCC identified severe AS in 76 patients, moderate AS in 30 patients, and mild AS in two patients. Relative to LCC PPSEPG, TTE MSEPG was found to be concordant in $79 / 108(81 / 108=73.1 \%)$. Of the 29 cases $(29 / 108=26.9 \%)$ of discordant data, TTE overestimated AS severity by $\geq 1$ grade in six patients $(6 / 108=5.6 \%)$. In contrast, TTE underestimated AS severity by $\geq 1$ grade in 23 patients $(23 / 108=21.3 \%)$. Importantly, because all patients met criteria for TAVI based upon pre-TAVI TTE determination of severe AS along with a consistent history and physical examination, TAVI was yet performed in patients in whom AS was assessed as not severe by either or both intra-procedural modalities. 
Table 2. Comparison of LCC and TTE assessments of AS. A. LCC-derived MSEPG and PPSEPG, compared to TTE-derived MSEPG. B. Relative concordance and discordance between LCC- and TTE-determined AS severity grade. C. True positive (both LCC and TTE demonstrating severe AS), true negative (both LCC and TTE demonstrating less-than-severe AS), false positive (LCC demonstrating less-than-severe AS, TTE demonstrating severe AS), and false negative (LCC demonstrating severe AS, TTE demonstrating less-than-severe AS) rates of TTE MSEPG versus LCC MSEPG; calculated sensitivity, specificity, positive predictive value (PPV), and negative predictive value (NPV) of TTE MSEPG at $\geq 40, \geq 35$ and $\geq 30 \mathrm{~mm} \mathrm{Hg}$ thresholds for severe AS.

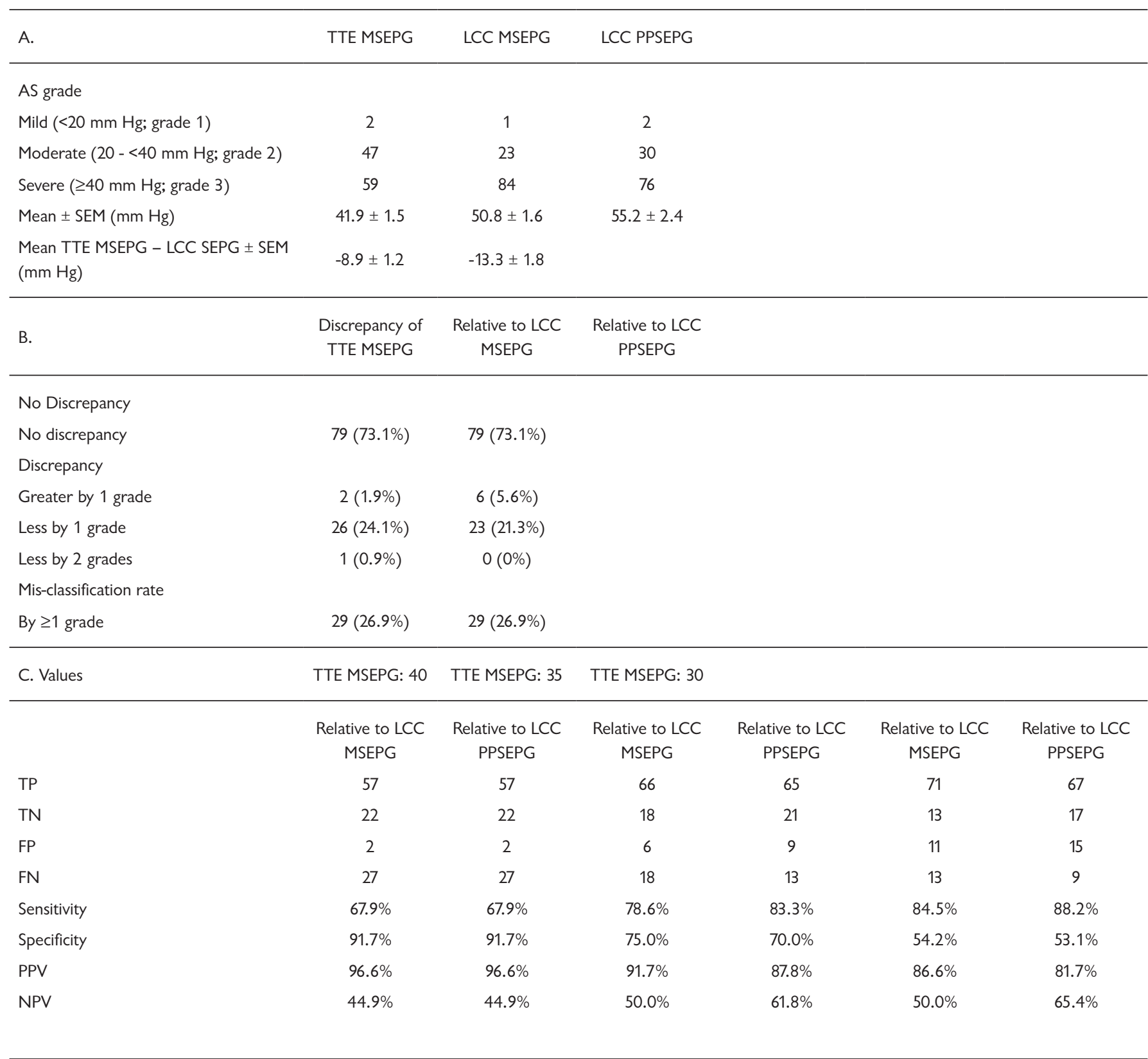

Effects of decreasing TTE threshold criteria on concordance with LCC: Because we observed underestimation of AS severity by TTE, we sought to ask whether lowering this threshold of TTE MSEPG of $\geq 40 \mathrm{~mm} \mathrm{Hg}$ for defining severe AS might result in improved correlation with LCC data. Therefore, reassessments were performed using a TTE MSEPG cutoff of $\geq 35$ or $\geq 30 \mathrm{~mm}$ $\mathrm{Hg}$ for defining severe AS. As shown in Table 2(C), the sensitivity of TTE using a $35 \mathrm{~mm} \mathrm{Hg}$ cutoff relative to LCC improved to $78.6 \%$ and $83.3 \%$, relative to MSEPG and PPSEPG, respectively; NPV increased as well, to $50.0 \%$ and $61.8 \%$, respectively. However, this was offset by reduced specificity and PPV. Specificity was reduced to $75.0 \%$ and $70.0 \%$, relative to LCC MSEPG and PPSEPG, respectively; PPV was reduced to $91.7 \%$ and $87.8 \%$, respectively. 
Receiver operator characteristic (ROC) relationships between TTE and LCC: ROC relationships between TTE MSEPG and LCC MSEPG as well as TTE MSEPG and LCC PPSEPG were derived as an additional approach to evaluating the quality of TTE assessments of AS (Figure 3). A value of LCC MSEPG of $\geq 40 \mathrm{~mm} \mathrm{Hg}$ was used as a gold standard to define severe AS. When TTE MSEPG was compared with LCC MSEPG, the area under the curve (AUC) was 0.842 , as depicted in Figure 3(A). Similarly, when TTE MSEPG was compared with LCC PPSEPG, the AUC was 0.868 , as shown in Figure 3(B). Furthermore, various cut-off values for TTE MSEPG of $\geq 30, \geq 35$ or $\geq 40 \mathrm{~mm} \mathrm{Hg}$ and their corresponding sensitivities have been included in these ROC curves. These data suggest that TTE exhibits a decent ability to identify severe AS as assessed by LCC. However, underestimation and significant misclassification of AS yet occurred by using TTE.
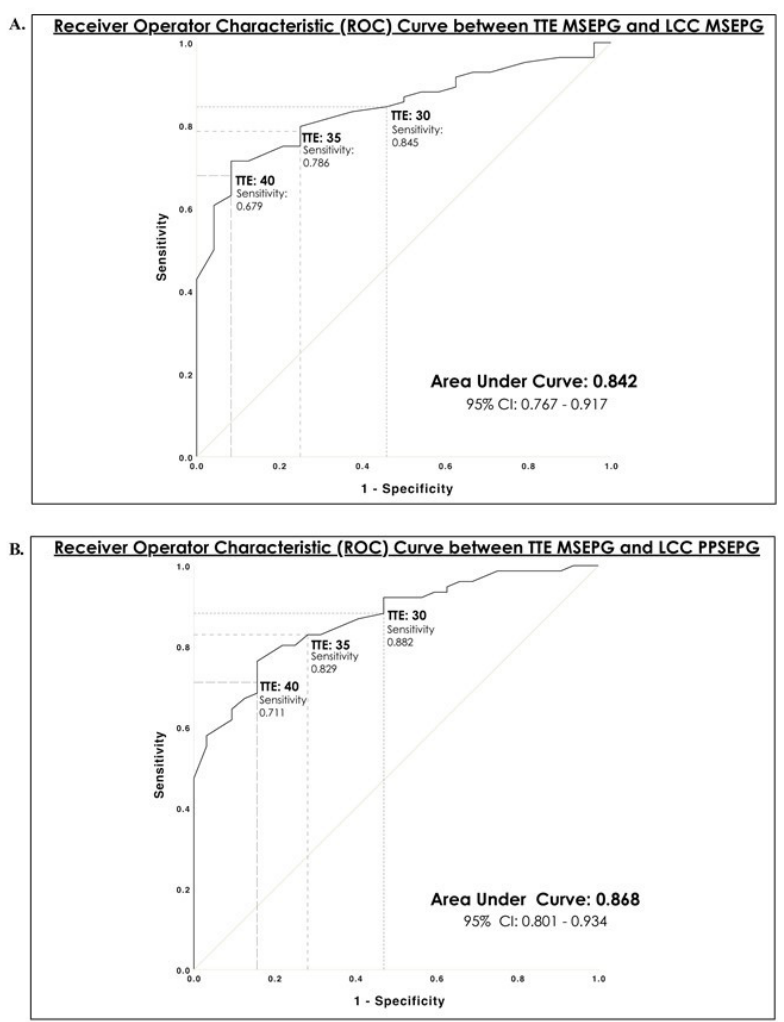

Figure 3. Diagnostic accuracy of intra-procedural TTE for AV assessment compared with LCC. Receiver operator characteristic (ROC) curves and corresponding areas under the curve (AUC) for various TTE cut-offs are shown. A) Comparison of TTE MSEPG (ROC) versus LCC MSEPG $(y=x)$. B) Comparison of TTE MSEPG (ROC) versus LCC PPSEPG $(y=x)$.

\section{DISCUSSION}

In this study, we conducted a retrospective comparison of invasively (LCC) and non-invasively (TTE) determined trans-AV systolic ejection pressure gradients, in the context of transfemoral TAVI undertaken in awake patients. Based upon our review of the literature, this appears to be the first such study in the setting of TAVI. We found that TTE commonly underestimates the MSEPG in a clinically important way. From a screening perspective, using non-standard lower TTE cutoffs improved sensitivity and NPV, albeit with reduced specificity and PPV. All patients met pre-procedural TTE criteria for undergoing TAVI, and thus subsequently thus underwent it regardless of the intra-procedural assessments (see discussion below). Thus, our data would suggest that if simultaneous LCC were hypothetically undertaken, even higher-pressure gradients would be identified.

However, if TTE underestimates SEPG in a clinically important way, then utilization of screening TTE to ascertain the severity of AS is called into question. Current TAVI criteria are reliant upon non-invasive assessments; this may be particularly problematic, as screening studies in principle should have low false-negative and high-sensitivity rates. Thus, it is plausible that a substantial percentage of patients undergoing isolated pre-TAVI (i.e., in a separate setting prior to TAVI, rather than intra-procedurally but pre-implantation) TTE assessments are denied AVR or TAVI that would be identifiably indicated if pre-TAVI LCC were undertaken. Our findings suggest that patients with other clinical data (history, physical examination, etc.) consistent with severe AS should undergo LCC assessment if TTE demonstrates the presence of moderate AS. However, based upon our study of lower TTE cutoff values, it is unclear as to what range of patients (or all) with moderate AS ought to undergo subsequent LCC.

We do not have information on patients who were screened by TTE and failed to demonstrate severe AS by echocardiographic criteria, only on those who qualified and underwent TAVI based upon these pre-procedural TTE assessments. In addition, because of potentially physiologically complex effects of MAC under which LCC is performed, outpatient TTE in an unsedated state cannot be compared with a temporally distinct LCC under MAC. Finally, resting TTE assessments may not reveal clinically important and hemodynamically significant AS, whereas studies under stressed conditions, in which systolic ejection trans-AV flow rates are higher, might reveal functionally severe AS [Johnson 2018].

If pre-procedural TTE findings are consistent with severe AS and intra-procedural LCC fails to confirm severe AS, this presents a different dilemma. All of the patients in our series had a pre-TAVI TTE identifying severe AS, but a substantial percentage of patients had intra-procedural LCC and TTE data that failed to identify at least moderate-severe AS $(24 / 108=22.2 \%$ for LCC, and $49 / 108=45.4 \%$ for TTE $)$. This highlights the high degree of variability in LV contractility and loading conditions between temporally distinct assessments, as well as potential operator dependence and other methodological variations between even the same techniques of assessments conducted at different times. However, 
because TTE predominantly appears to underestimate AS severity, our data suggest that if a TTE assessment identifies severe AS, it is probably reasonable to proceed with AVR or TAVI. As previously discussed, it is not possible to fairly compare a pre-procedural TTE with a potential pre-procedural LCC because the latter is performed under conscious sedation (MAC), whereas the former is not.

Important limitations exist in our study. It is a retrospective review of a single interventional cardiologist's experience. With respect to methodology, although LCC is the gold standard for assessment of AS severity because pressure data are measured, the presence of even a small bore catheter spanning the AV orifice may exacerbate severe AS (Carabello effect) [Carabello 1979], even more so when AS is critical. However, the cross-sectional area of the LV pressure-transducing catheters in this study are only $\sim 3.1 \mathrm{~mm}^{2}$, much smaller than AV orifice areas even in critical AS. Pre-procedural TTE data is not being reported. PISEPG data were not obtained during LCC; thus, we were unable to compare LCC- and TTEderived PISEPG. In addition, operator dependence with respect to TTE assessments is well-described [Sacchi 2018]; it is conceivable that TTE performed at our institution consistently underestimated AS severity.

In LCC, pressure data are measured directly; valve resistance can be calculated if the cardiac output, and thus stroke volume, are known. In contrast, non-invasive imaging techniques, most notably echocardiography, rely upon approaches to calculate pressure. It has been shown [Svihlova 2017; Svihlova 2016] that standard non-invasive approaches for calculating transvalvular pressure differences have both conceptual and methodological drawbacks (Figure 1; see below). Moreover, using state-of-the-art fluid mechanics theory, solving inverse problems in the Navier-Stokes equations [Svihlova 2016], rigorous approaches to ascertaining LV pressure, intra- $\mathrm{AV}$ orifice velocity, and energy dissipation, from pre-specified LV outflow tract velocity and proximal aortic pressure data, have been developed [Svihlova 2017].

Currently used non-invasive approaches to calculating transvalvular pressure gradients employ the Bernoulli equation. However, the Bernoulli equation assumes inviscid flow, i.e., zero blood viscosity in the context of blood flow, and expresses conservation of energy. Viscosity is the physical property of fluids that is necessary for energy losses (dissipation) that occur with flow. Consequently, if the Bernoulli equation is invoked - expressing energy-conservative inviscid flow - concepts expressing energy-dissipative flow such as "resistance" and for time-varying (e.g., pulsatile) flow, "impedance," are zero. The defining characteristic of a stenosis is that it poses an impedance to flow, i.e., fluid energy is lost due to energy dissipation across the stenosis. There is thus only one circumstance under which the Bernoulli equation can be appropriately applied to a stenosis in order to determine its hemodynamic importance. This is if the pressure within the stenosis happens to be equal to the pressure distal to the stenosis. The entirety of energy losses of flowing blood must occur just distal to the stenosis, where cross-sectional area acutely increases, and are mathematically captured in toto by losses in blood kinetic energy in the immediate post-stenotic segment (although, as forms of energy are equivalent and interconvertible, this is simply an "accounting" tool with respect to mathematically expressing energy losses). In this situation, even though the Bernoulli equation still does not actually hold in that energy is dissipated, it can be used to calculate pressure gradients if the velocity data are known. However, the aforementioned assumptions are not wellsupported. So-called "pressure recovery," in which the pressure distal to the stenosis is higher than that within the stenosis, is impermissible if the circumstances above are invoked, but commonly occurs in vivo in the setting of valvular stenoses [Rijsterborgh 1987].

While it might be hypothesized that TTE should systematically overestimate LCC-derived pressure gradients (the lack of conceptual basis for this notion is described in the Figure 1 Legend), there is substantial variability in the literature regarding correlation between LCC- and TTE-derived data. Some of the earliest studies [Stamm 1983; Currie 1985] demonstrated good correlation between LCC and TTE, although a degree of underestimation via TTE was identified; yet, TTE appeared to have a low rate of mis-classification of AS severity in these studies. However, as previously noted, Oh et al [Oh 1988] identified large discrepancies between TTE and LCC, with clinically relevant mis-classification of AS severity by TTE. In addition, larger and/or more recent studies have identified less predictable and more complex non-linear, albeit yet roughly proportional, relationships between LCC- and TTE-determined assessments [Otto 1992; Parameswaran 2009].

Finally, it remains unknown whether the clinical importance of AS is in fact related to physiological stenosis severity, as opposed to simply anatomic severity. It is well-known in fluid mechanics that the relationship between conduit cross-sectional area and energy losses is non-linear; as such, anatomically significant stenoses that do not result in substantial energy dissipation may exist. However, it is possible that clinically important sequelae of AS are not only related to energy dissipation, but simply the anatomic extent of AS. For example, a non-dissipative stenosis yet could be associated with low aortic root and thus coronary arterial pressures, thereby resulting in impaired myocardial perfusion and resultant ischemia.

In our view however, the principal aim and impact of this study relates to clinical practice, regardless of potential conceptual advantages to LCC versus non-invasive approaches. Because TAVI criteria are based upon non-invasive modalities, inaccuracies in these modalities warrant either invasive assessment, or refinement of non-invasive criteria. Future studies are essential in order to better delineate both of these diagnostic options.

\section{ACKNOWLEDGMENTS}

We thank Hang Duong and Melissa Howe for assistance with data collection. This study was funded by internal University of Texas-Houston departmental funding. PL, ALE, $\mathrm{HJS}$, and KR are institutional investigators or subinvestigators 
on Edwards Lifesciences-sponsored clinical TAVI trials. PL is an uncompensated proctor for Edwards Lifesciences.

\section{REFERENCES}

Adams DH, Popma JJ, Reardon MJ, et al. 2014. U.S. CoreValve Clinical Investigators. Transcatheter aortic valve replacement with a self-expanding prosthesis. New Engl J Med. 370(19):1790-1798.

Andell P, Li X, Martinsson A, et al. 2017. Epidemiology of valvular heart disease in a Swedish nationwide hospital-based register study. Heart. 103(21):1696-1703.

Braunwald E. 2018. Aortic stenosis: then and now. Circulation. May 15;137(20):2099-2100.

Carabello BA, Barry WH, Grossman W. 1979. Changes in arterial pressure during left heart pullback in patients with aortic stenosis: a sign of severe aortic stenosis. Am J Cardiol. 44(3):424-427.

Currie PJ, Seward JB, Reeder GS, et al. 1985. Continuous-wave Doppler echocardiographic assessment of severity of calcific aortic stenosis: a simultaneous Doppler-catheter correlative study in 100 patients. Circulation. 71(6):1162-1169.

Ford LE, Feldman T, Chiu YC, Carroll JD. 1990. Hemodynamic resistance as a measure of functional impairment in aortic valvular stenosis. Circ Res. 66(1):1-7.

Gertz ZM, Raina A, O’Donnell W, et al. 2012. Comparison of invasive and non-invasive assessment of aortic stenosis severity in the elderly. Circ Cardiovasc Interv. 5(3): 406-414.

Johnson NP, Zelis JM, Tonino PAL, et al. 2018. Pressure gradient vs. flow relationships to characterize the physiology of a severely stenotic aortic valve before and after transcatheter valve implantation. Eur Heart J. 39(28): 2646-2655.

Lauck SB, Wood DA, Achtem L, et al. 2014. Risk stratification and clinical pathways to optimize length of stay after transcatheter aortic valve replacement. Can J Cardiol. 30(12):1583-1587.

Leon MB, Smith CR, Mack M, et al. 2010. PARTNER Trial Investigators. Transcatheter aortic-valve implantation for aortic stenosis in patients who cannot undergo surgery. New Engl J Med. 363(17):1597-1607.

Oh JK, Taliercio CP, Holmes Jr DR, et al. 1988. Prediction of the severity of aortic stenosis by Doppler aortic valve area determination: prospective Doppler-catheterization correlation in 100 patients. J Am Coll
Cardiol. 11(6): 1227-1234.

Otto CM, Davis KB, Holmes DR Jr, et al. 1992. Methodologic issues in clinical evaluation of stenosis severity in adults undergoing aortic or mitral balloon valvuloplasty. The NHLBI Balloon Valvuloplasty Registry. Am J Cardiol. 69(19):1607-1616.

Otto CM, Kumbhani DJ, Alexander KP, et al. 2017. 2017 ACC expert consensus decision pathway for transcatheter aortic valve replacement in the management of adults with aortic stenosis: a report from the American College of Cardiology Taskforce on Clinical Expert Consensus Documents. J Am Coll Cardiol. 69(10): 1313-1346.

Parameswaran AC, Reisner D, Amanullah A. 2009. Discrepancy between gradients derived by cardiac catheterization and by Doppler echocardiography in aortic stenosis: how often does pressure recovery play a role? Echocardiography. 26(9):1000-1005.

Popma JJ, Adams DH, Reardon MJ, et al. 2014. CoreValve United States Clinical Investigators. Transcatheter aortic valve replacement using a self-expanding bioprosthesis in patients with severe aortic stenosis at extreme risk for surgery. J Am Coll Cardiol. 63(19):1972-1981.

Reineke D, Gisler F, Englberger L, Carrel T. 2016. Mechanical versus biological aortic valve replacement strategies. Expert Rev Cardiovasc Ther. 14(4):423-430.

Rijsterborgh H, Roelandt J. 1987. Doppler assessment of aortic stenosis: Bernoulli revisited. Ultrasound Med Biol. 13(5):241-248.

Sacchi S, Dhutia NM, Shun-Shin MJ, et al. 2018. Doppler assessment of aortic stenosis: a 25-operator study demonstrating why reading the peak velocity is superior to the velocity-time integral. Eur Heart J Cardiovasc Imaging. e-pub ahead of print.

Smith CR, Leon MB, Mack MJ, et al. 2011. PARTNER Trial Investigators. Transcatheter versus surgical aortic-valve replacement in high-risk patients. New Engl J Med. 364(23):2187-2198.

Stamm RB, Martin RP. 1983. Quantification of pressure gradients across stenotic valves by Doppler ultrasound. J Am Coll Cardiol. 2(4):707-718.

Svihlova H, Hron J, Malek J, Rajagopal KR, Rajagopal K. 2016. Determination of pressure data from velocity with a view toward its application in cardiovascular mechanics. Part 1 . Theoretical considerations. Int J Eng Sci. 105(1): 108-127.

Svihlova H, Hron J, Malek J, Rajagopal KR, Rajagopal K. 2017. Determination of pressure data from velocity with a view toward its application in cardiovascular mechanics. Part 2. A study of aortic valve stenosis. Int J Eng Sci. 114(1):1-15. 